\title{
Calcium-Dependent Protein Kinase Family Genes Involved in Ethylene-Induced Natural Rubber Production in Different Hevea brasiliensis Cultivars
}

\author{
Liping Zhu ${ }^{1,+}$, Xiang Jin ${ }^{1,2,3,+}$, Quanliang Xie ${ }^{1}$, Qi Yao ${ }^{2}$, Xuchu Wang ${ }^{1,2, *(\mathbb{D})}$ and Hongbin Li ${ }^{1, *}$ \\ 1 College of Life Sciences, Key Laboratory of Xinjiang Phytomedicine Resource Utilization of Ministry of Education, \\ Shihezi University, Shihezi 832003, China; zhuliping0903@163.com (L.Z.); jinxiang@itbb.org.cn (X.J.); \\ xiequanliang001@163.com (Q.X.) \\ 2 College of Life Sciences, Ministry of Education Key Laboratory for Ecology of Tropical Islands, \\ Hainan Normal University, Haikou 571158, China; yaoqi7@outlook.com \\ 3 Institute of Tropical Biosciences and Biotechnology, Chinese Academy of Tropical Agricultural Sciences, \\ Haikou 571101, China \\ * Correspondence: xchwanghainan@163.com (X.W.); lihb@shzu.edu.cn (H.L.); Tel.: +86-898-6698-7460 (X.W.); \\ +86-993-205-7912 (H.L.) \\ + These authors contributed equally to this work.
}

\begin{abstract}
Natural rubber latex production can be improved by ethylene stimulation in the rubber tree (Hevea brasiliensis). However, the expression levels of most functional proteins for natural rubber biosynthesis are not induced after ethylene application, indicating that post-translational modifications, especially protein phosphorylation, may play important roles in ethylene signaling in Hevea. Here, we performed a comprehensive investigation on evolution, ethylene-induced expression and protein-protein interaction of calcium-dependent protein kinases (CPKs), an important serine/threonine protein kinase family, in Hevea. Nine duplication events were determined in the 30 identified $H b C P K$ genes. Expression profiling of $H b C P K s$ in three rubber tree cultivars with low, medium and high ethylene sensitivity showed that $H b C P K 6,17,20,22,24,28$ and 30 are induced by ethylene in at least one cultivar. Evolution rate analysis suggested accelerated evolution rates in two paralogue pairs, $H b C P K 9 / 18$ and $H b C P K 19 / 20$. Analysis of proteomic data for rubber latex after ethylene treatment showed that seven $\mathrm{HbCPK}$ proteins could be detected, including six ethylene-induced ones. Protein-protein interaction analysis of the 493 different abundant proteins revealed that protein kinases, especially calcium-dependent protein kinases, possess most key nodes of the interaction network, indicating that protein kinase and protein phosphorylation play important roles in ethylene signaling in latex of Hevea. In summary, our data revealed the expression patterns of $H b C P K$ family members and functional divergence of two $H b C P K$ paralogue pairs, as well as the potential important roles of $\mathrm{HbCPKs}$ in ethylene-induced rubber production improvement in Hevea.
\end{abstract}

Keywords: Hevea brasiliensis; calcium-dependent protein kinase; gene family evolution; ethylene signaling; protein-protein interaction network

\section{Introduction}

Rubber tree (Hevea brasiliensis) is mainly cultivated in the tropical regions, providing the most production of natural rubber, an important industrial material [1]. Ethylene is applied as a routine stimulant to increase the rubber latex yield of rubber tree in agricultural practice for years [2]. However, the molecular mechanism of ethylene signaling of rubber production improvement is not clarified. 
Despite the difficulty of molecular genetic research for rubber tree, many works have been performed to investigate important factors in ethylene-induced rubber production improvement (EIRP). Several physiological processes, such as sucrose transport [3], water uptake [4], and synthesis of defense proteins [5] are reported to be activated after ethylene stimulation in $\mathrm{H}$. brasiliensis (termed as Hevea hereafter). Many functional genes have been identified as to be regulated by ethylene. Sucrose transporter $1 \mathrm{~A}$ and sucrose transporter 2A can increase sucrose import into laticifers after ethylene stimulation and further increase latex yield [6]. Gene expression analysis showed that ethylene receptor 1, ethylene receptor 2, ethylene insensitive protein 2, and plasma membrane aquaporin were specifically regulated by ethylene treatment [7]. In addition, microRNAs, miR167 (targeting auxin response factor 6) and miR172 (targeting rubber elongation factor 3) were reported to play potential roles in response to ethylene treatment and further improve the rubber latex yield [8]. For the aspect of protein level, various functional proteins involved in natural rubber biosynthesis are not induced upon ethylene treatment, which implies the post-translational modification might be important for EIRP [9]. Our previous work revealed that protein phosphorylation might be important for EIRP [10]. Thus, investigation of protein kinases and the protein phosphorylation catalyzed by kinases in rubber tree will be helpful to better understand the molecular mechanism of EIRP.

Calcium-dependent protein kinase (CPK) belongs to serine/threonine protein kinase family, which contains four domains: N-terminal variable domain, serine/threonine kinase domain, auto-inhibitory junction domain and EF-hand calcium binding domain [11-13]. N-terminal variable domain is highly variable in both length and sequences, and contains myristoylation and palmitoylation sites, which contribute to membrane localization [12]. Auto-inhibitory domain serves as a pseudo-substrate and blocks the activity of the enzyme in the absence of $\mathrm{Ca}^{2+}$ stimulation with CaM-LD binding site [14]. The EF-hand calcium binding domains hold up four elongation factor hands and CPKs in monocot and dicot plants possess at least four D-x-D and two D/E-E-L motifs in their EF-hands, that bind the calcium icons and play a significant role in gene regulation [12,14]. Depending on the unique structure, a kinase domain with an EF-hand containing regulatory domain, CPKs can respond diverse endogenous and environmental cues and translate $\mathrm{Ca}^{2+}$ signals into downstream protein phosphorylation [15]. CPKs play important roles in many aspects of plant growth and development and in responding to various stimulate including biotic and abiotic stresses and hormones [16].

Genome-wide investigation of CPK gene family have been reported in Arabidopsis (Arabidopsis thaliana), rice (Oryza sativa), soybean, maize and grape, as well as cassava (Manihot esculenta), which is closely related to Hevea [12,16-20]. The HbCPK gene family has been reported recently, identifying $30 \mathrm{HbCPKs}$ and profiling their expression patterns in six plant species using next-generation sequencing data [21]. However, the evolutionary and syntenic relationships of $H b C P K$ members, as well as the protein expression levels of CPK family members in response to ethylene treatment were not thoroughly analyzed. Here, we performed a comprehensive evolutionary and syntenic analysis of $H b C P K$ genes. The expression profiles of $H b C P K s$ in different tissues and after ethylene treatment in three varieties were analyzed by quantitative real-time PCR (qRT-PCR) and semi-quantitative PCR. The protein-protein interaction (PPI) network of ethylene-response proteins were further analyzed, suggesting that the kinase protein members and phosphorylated proteins were involved in the key nodes of the ethylene-induced protein interaction network clusters. Our work provides a more comprehensive investigation of the evolutionary relationships, expression patterns and PPI relationships of CPK members in ethylene signaling in Hevea, as well as potential transgenic targets of molecular breeding of rubber tree for improving natural rubber production in the future.

\section{Results}

\subsection{Identification and Characteristics of CPK Members in H. brasiliensis}

A BLASTP search for Hevea genome database was performed using Arabidopsis and rice CPK protein sequences, a total of 41 genome-deduced proteins were identified as $\mathrm{HbCPK}$ candidates. 
Only sequences containing both protein kinase domain (PF00069) and EF-hand_7 (PF13499) domain were confirmed as CPKs, resulting in $30 \mathrm{HbCPK}$ family members (Table S1). The open reading frame lengths of the 30 identified $H b C P K s$ range from $1308 \mathrm{bp}$ to $1896 \mathrm{bp}$, and the calculated protein molecular weights range from 49.19 to $70.79 \mathrm{kDa}$ and isoelectric points range from 5.14 to 9.25 .

The $30 \mathrm{HbCPK}$ members were distributed onto 30 different scaffolds (Figure S1). Nine duplicated events were identified (HbCPK1/HbCPK26, HbCPK2/HbCPK7, НbCPK3/HbCPK8, HbCPK4/HbCPK11,

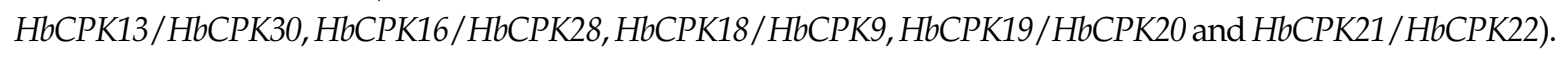
The conserved domains were shown in multiple sequence alignment of $\mathrm{HbCPKs} \mathrm{(Figure} \mathrm{S2).} \mathrm{In} \mathrm{addition,}$ 12 conserved motifs were predicted using Multiple Em for Motif Elicitation (MEME, version 4.12.0) (http://meme-suite.org/doc/authors.html?man_type=web) program (Figure S3). Motif 3, 7, 5, 8 and 9 represent protein kinase domain, auto-inhibitory domain and four EF-hand domains, respectively. A phylogenetic tree was constructed using the CPK protein sequences of Arabidopsis, O. sativa, M. esculenta and Hevea, dividing the CPKs into four groups (Group I to IV), containing eleven, eight, eight and two members, respectively (Figure S4).

\subsection{Gene Structure, Duplication Events and Syntenic Analysis}

The exon-intron distribution reveals the evolutionary relationship of gene families [22]. Gene structure analysis of $\mathrm{HbCPKS}$ showed that the members in same subfamily shared similar distribution pattern in both numbers and length of exons and introns. As shown in Figure 1, group I contains 6-7 exons, group II contains 7-12 exons, group III contains 7-8 exons and group IV contains 12 exons. Moreover, the paralogue pairs of HbCPK3/HbCPK8, HbCPK13/HbCPK30, HbCPK18/HbCPK9 and $\mathrm{HbCPK19/HbCPK20}$ were determined as rubber tree specific duplicated events by comparing the phylogenetic relationship and exon-intron distribution patterns of $H b C P K s$ and that of MeCPKs.

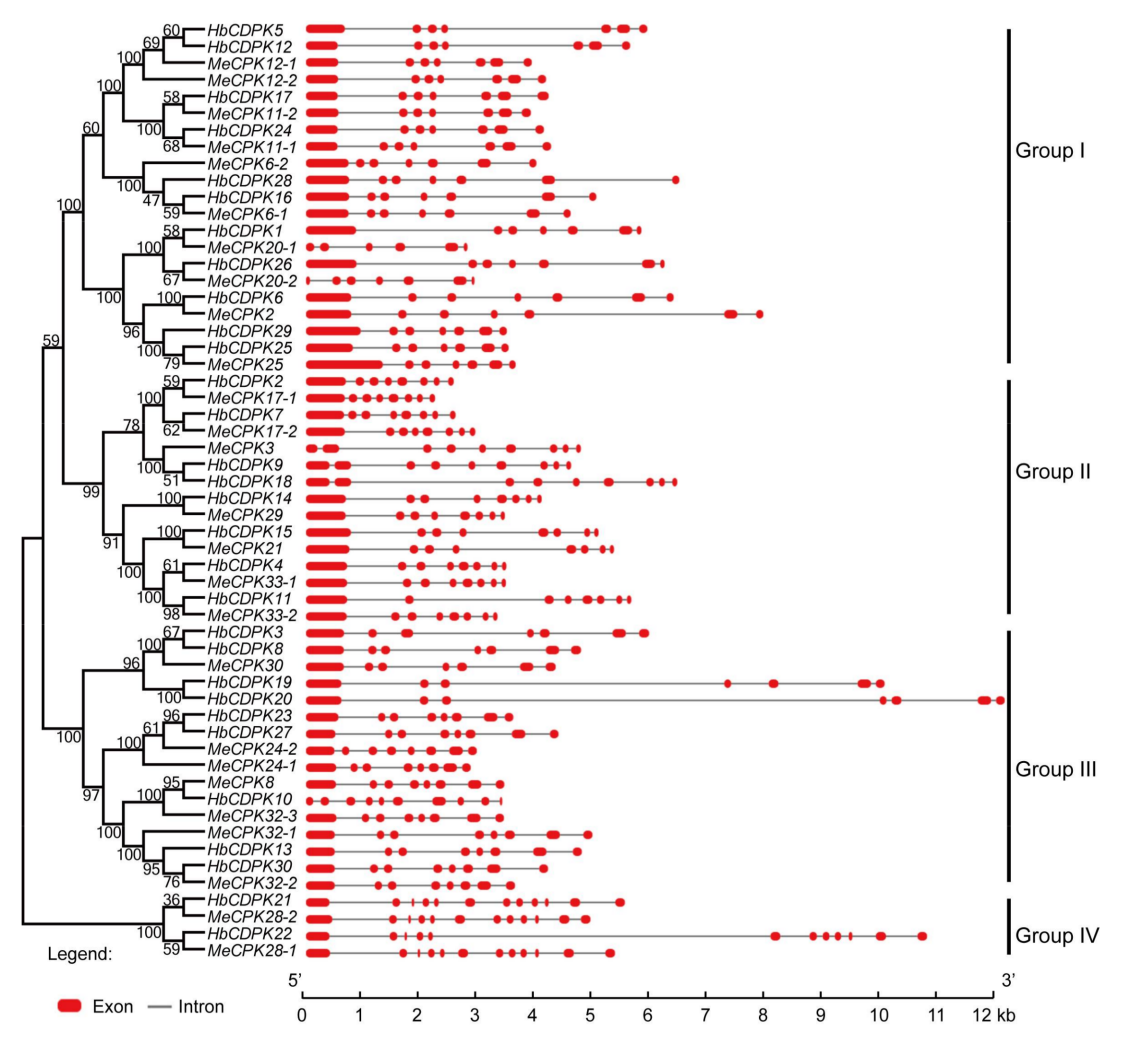

Figure 1. Phylogenetic and gene structure analysis of $C P K s$. Exon-intron distribution was analyzed using GSDS software 2.0 (http://gsds.cbi.pku.edu.cn/). Tree-view is on the left, exon-intron distribution is on the right side, introns and exons are represented by black lines and red boxes, respectively, and the genomic length is indicated at the bottom. 
The syntenic analysis of Hevea was performed using gene location information of CPKs in Arabidopsis and a closely related spurge plant cassava to investigate the expansion of $H b C P K$ family. No tandem duplication events were discovered because of the limitation of chromosomal information of the current version of rubber tree genome assembly. Nine segmental duplication events were identified and the paralogue gene pairs were then defined as syntenic genes (Figure 2).

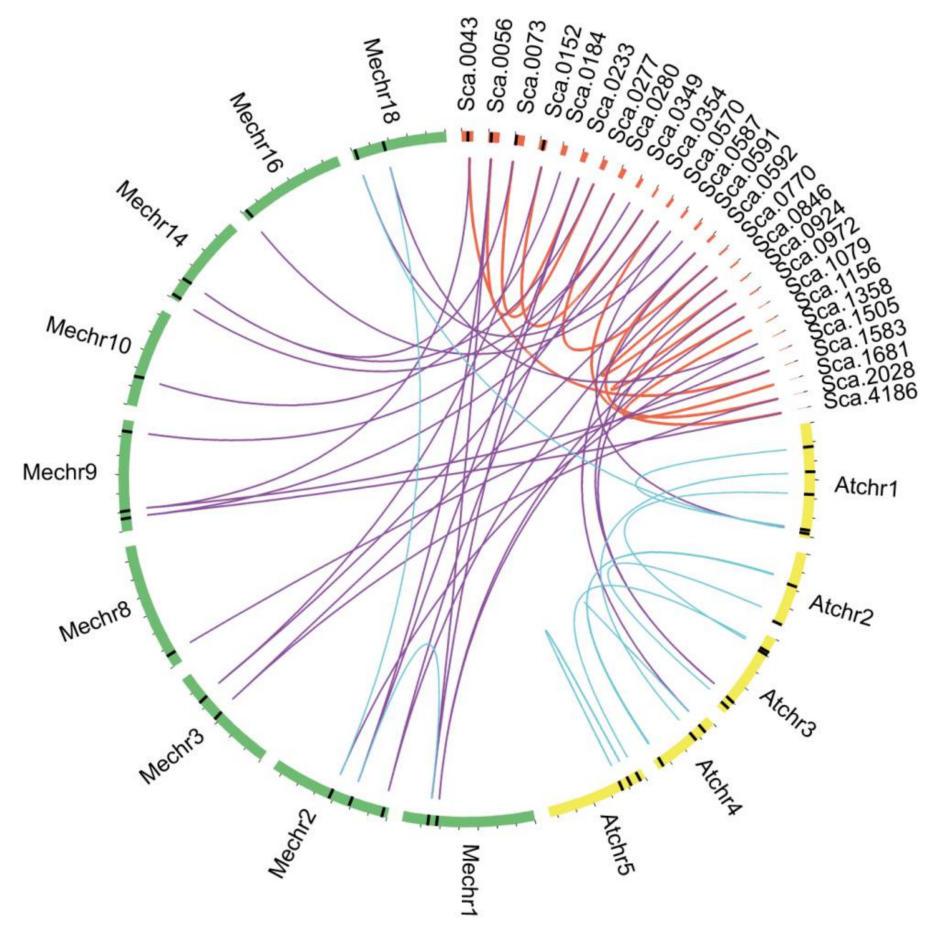

Figure 2. Segmental duplication of $H b C P K$ genes and syntenic analysis of $H$. brasiliensis, M. esculenta and A. thaliana CPKs. Chromosomes and scaffolds are shown in different colors in circular form. The positions of the CPK genes are marked with black lines on the circle. The duplicated CPK pairs in $H$. brasiliensis are linked by red lines and syntenic relationships between $H$. brasiliensis and the other two species are linked by purple lines, while blue lines indicate the $C P K$ pairs between or inside $M$. esculenta and A. thaliana.

The $\mathrm{Ka} / \mathrm{Ks}$ ratio was calculated to assess the selection pressure for each duplicated $\mathrm{HbCPK}$ paralogue pairs. The results showed that all paralogues were under purifying selection pressure (Table 1). Meanwhile, Tajima relative rate was used to estimate the evolutionary rate of these duplication pairs. The $H b C P K 9 / H b C P K 18$ and $H b C P K 19 / H b C P K 20$ duplication pairs have a prominently accelerate evolutionary rates (Table 2).

Table 1. The $\mathrm{Ka} / \mathrm{Ks}$ ratios for duplicate CPK genes in $\mathrm{H}$. brasiliensis.

\begin{tabular}{ccccl}
\hline Paralogous Genes & $\boldsymbol{K} \boldsymbol{a}$ & $\boldsymbol{K} \boldsymbol{s}$ & $\boldsymbol{K a} / \mathbf{K s}$ & Selective Pressure \\
\hline HbCPK16-HbCPK28 & 0.0129 & 0.1660 & 0.0777 & Purifying selection \\
HbCPK1-HbCPK26 & 0.0276 & 0.2004 & 0.1377 & Purifying selection \\
HbCPK3-HbCPK8 & 0.0193 & 0.2290 & 0.0843 & Purifying selection \\
HbCPK13-HbCPK30 & 0.0274 & 0.1840 & 0.1489 & Purifying selection \\
HbCPK4-HbCPK11 & 0.0308 & 0.2527 & 0.1219 & Purifying selection \\
HbCPK9-HbCPK18 & 0.0434 & 0.1876 & 0.2313 & Purifying selection \\
HbCPK2-HbCPK7 & 0.0227 & 0.1941 & 0.1170 & Purifying selection \\
HbCPK21-HbCPK22 & 0.0169 & 0.2004 & 0.0843 & Purifying selection \\
HbCPK19-HbCPK20 & 0.0214 & 0.1767 & 0.1211 & Purifying selection \\
\hline
\end{tabular}

Ka: non-synonymous substitution rate; Ks: synonymous substitution rate. 
Table 2. Tajima relative rate tests of $C P K$ gene pairs in rubber tree ${ }^{\mathrm{a}}$.

\begin{tabular}{cccccc}
\hline Testing Group & $\mathbf{M t}^{\mathbf{b}}$ & $\mathbf{M 1}^{\mathbf{c}}$ & $\mathbf{M 2}^{\mathbf{d}}$ & $\mathbf{X}^{\mathbf{2}}$ & $\boldsymbol{P}^{\mathbf{e}}$ \\
\hline HbCPK4/HbCPK11 with MeCPK33-2 & 489 & 20 & 13 & 1.48 & 0.22302 \\
HbCPK9/HbCPK18 with MeCPK3 & 457 & 7 & 47 & 29.63 & 0.00001 \\
HbCPK16/HbCPK28 with MeCPK6-1 & 529 & 8 & 7 & 0.07 & 0.79625 \\
HbCPK26/HbCPK1 with MeCPK20-1 & 250 & 5 & 11 & 2.25 & 0.13361 \\
HbCPK3/HbCPK8 with MeCPK30 & 514 & 7 & 16 & 3.52 & 0.06057 \\
HbCPK30/HbCPK13 with MeCPK32-2 & 485 & 10 & 17 & 1.81 & 0.17793 \\
HbCPK19/HbCPK20 with MeCPK30 & 370 & 6 & 0 & 6.00 & 0.01431 \\
HbCPK2/HbCPK7 with MeCPK17-2 & 465 & 16 & 9 & 1.96 & 0.16151 \\
HbCPK22/HbCPK21 with MeCPK28-1 & 507 & 17 & 10 & 1.81 & 0.17793 \\
\hline
\end{tabular}

\footnotetext{
a the Tajima relative rate test was used to examine the equality of evolutionary rate between rubber tree paralogues; b, Mt is the sum of the identical sites in all three sequences tested; ${ }^{c}, \mathrm{M} 1$ is the number of unique differences in the first paralog; ${ }^{\mathrm{d}}, \mathrm{M} 2$ is the number of unique differences in the second paralog; ${ }^{\mathrm{e}}$, if $p<0.05$, the test rejects the equal substitution rates between the two duplicates and infers that one of the two duplicates has an accelerated evolutionary rate.
}

\subsection{Tissue-Specific and Ethylene-Induced Expression Analysis of HbCPKs in Three Rubber Tree Varieties}

Root, stem, leaf and latex of three cultivated rubber tree varieties (Reyan8-79 with high yielding, low ethylene sensitivity; Reyan7-33-97 with medium yielding and medium ethylene sensitivity; PR107 with low yielding and high ethylene sensitivity) were used in this study to provide better understanding of the roles of $H b C P K s$. The expression pattern of $H b C P K s$ was visualized using qRT-PCR (Figure 3A) and semi-quantitative PCR assays (Figure 3B) to analyze the tissue specific expressions. Gene-specific primers used in PCR assays are listed in Table S2. The expression pattern can be divided into three clusters: $H b C P K 12,13,21,22,24$ and 28 of cluster TI mainly expressed in all four tissues; $H b C P K 1,3,4,5,6,8,9,11,15,16,18,19,20$, and 30 of cluster TII mainly expressed in roots, stems and leaves of Reyan7-33-97 and Reyan8-79; HbCPK2, 7, 14, 17, 23, 25, 26, 27 and 29 of cluster TIII could not be detected. The members of HbCPK12, HbCPK13, HbCPK21, HbCPK22, HbCPK24 and $\mathrm{HbCPK} 28$ showed high expression levels in latex, indicating their potential function in rubber latex production. Notably, paralogue pairs with accelerated evolutionary rates showed divergent expression patterns in different tissues (HbCPK9/HbCPK18 and HbCPK19/HbCPK20), suggesting their potential functional divergence after duplication events (Figure 3).

The expression levels of $\mathrm{HbCPKs}$ were further examined in the latex of three cultivars after ethylene treatment for $0,3,12$ and $24 \mathrm{~h}$. The results showed that $H b C P K 13, H b C P K 21$ and $H b C P K 28$ were significantly induced after ethylene stimulation in all three cultivars. Meanwhile, the expression levels of $H b C P K 20, H b C P K 22$ and $H b C P K 30$ were specifically promoted by ethylene stimulation in the ethylene-sensitive cultivar PR107 (Figure 4). Moreover, HbCPK6 and HbCPK30 which have very low expression levels in latex before ethylene treatment showed significantly up-regulated after ethylene stimulation.

\subsection{Cis-Element Distribution Analysis of Promoter Regions in HbCPKs}

The distribution of cis-elements in the promoter regions of $H b C P K s$ was analyzed to investigate the potential regulation factors of $H b C P K$ family members. The cis-elements were symbolized by capital letters with different colors and detailed information was also supplied (Figure 5, Table S3). The results showed that the promoter regions in HbCPK1, HbCPK2, HbCPK4, HbCPK7, HbCPK18 and $\mathrm{HbCPK} 26$ have one or two ethylene response cis-elements, suggesting the potential regulation of these genes under ethylene. Notably, the cis-element distribution in promoters of paralogues $\mathrm{HbCPK9/18}$ and $H b C P K 19 / 20$ with accelerated evolutionary rates are various, providing further evidence that these two paralogue pairs have different regulation patterns and are ongoing functional divergence. 


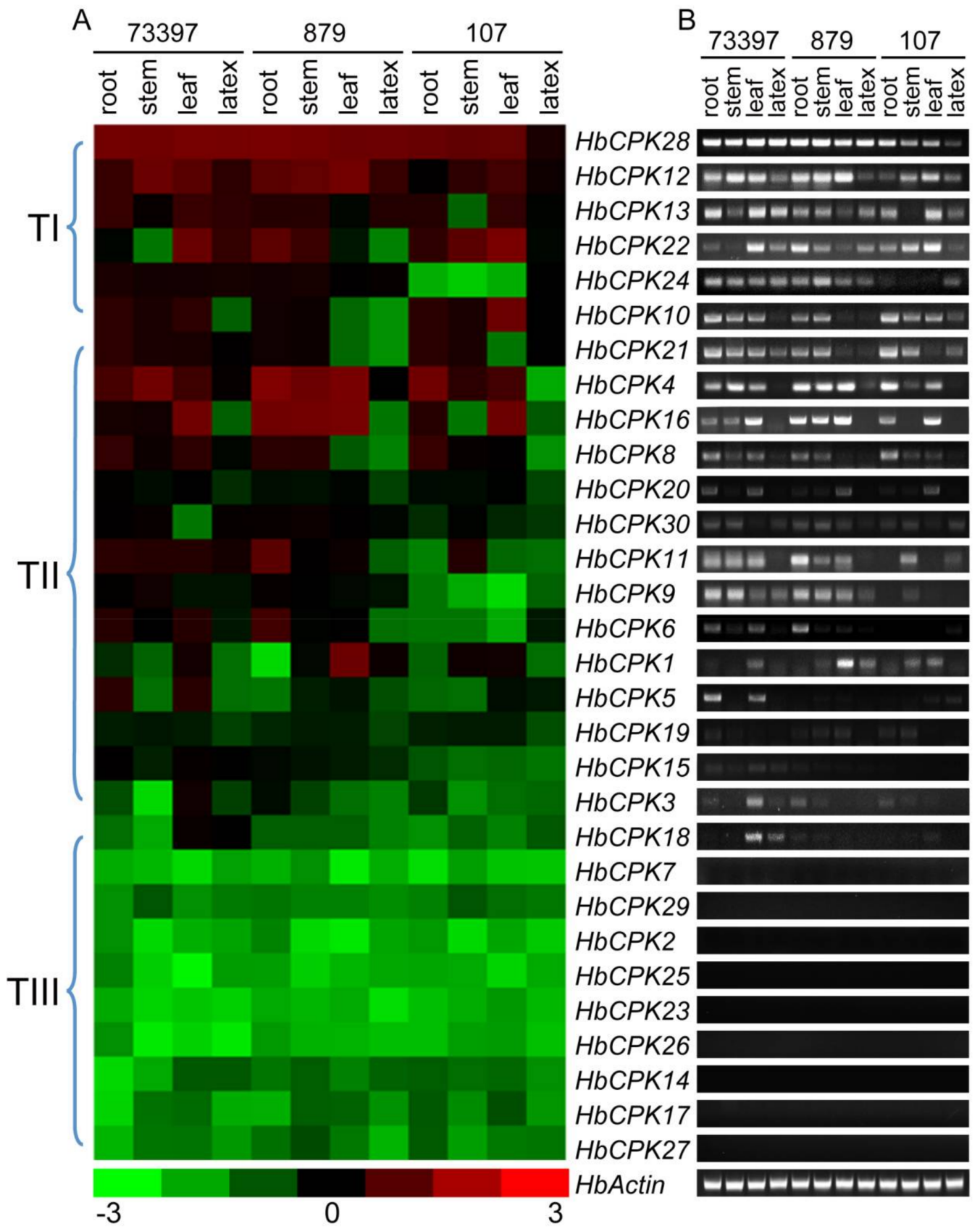

Figure 3. Expression profiles of $H b C P K s$ in different tissues obtained from three cultivars. Expression patterns of $H b C P K s$ were determined by qRT-PCR (A) and semi-quantitative PCR (B). The HbCPK genes were clustered by their expression patterns (left). HbActin was used as reference gene. The semi-quantitative PCR results were shown in electrophoretogram (B). 
A

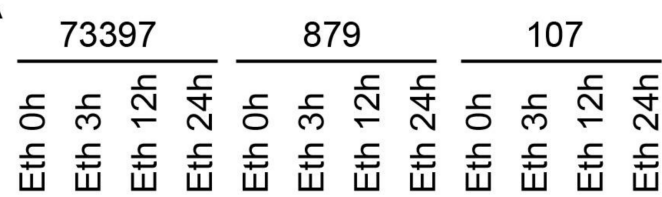

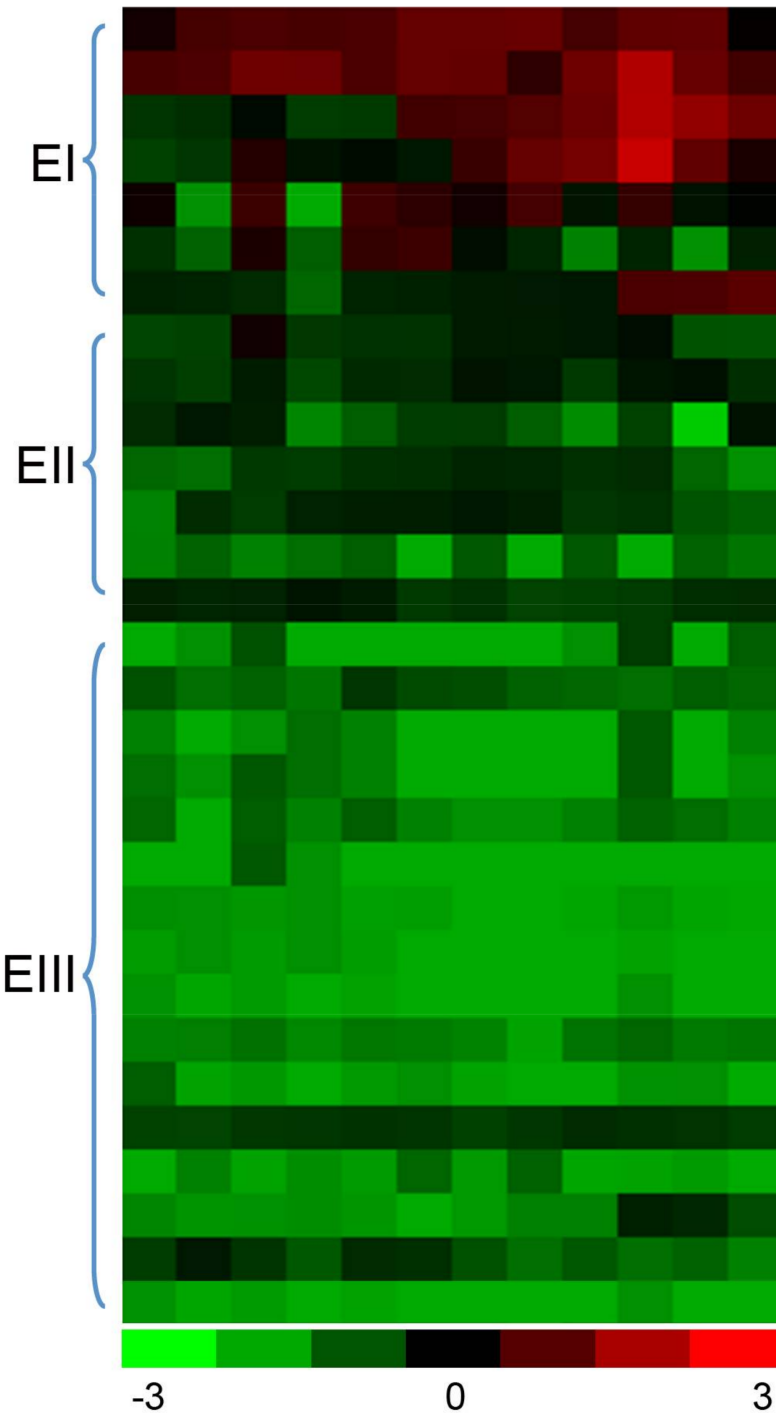

B

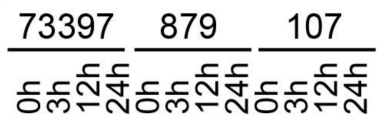

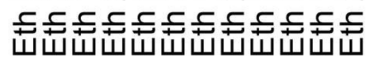

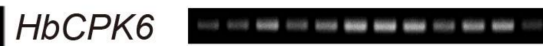

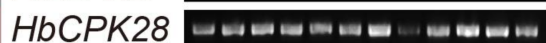
HbCPK22 드므브브브

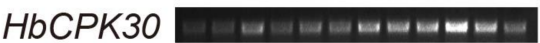
$\mathrm{HbCPK} 24$ [ $=-=$ HbCPK17 $\square=-\square=0$

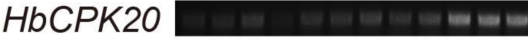
HbCPK13 ㅁaㅁㅁaaa․

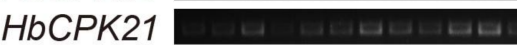
HbCPK1 $\mathrm{HbCPK8}$ $\mathrm{HbCPK3}$ $\mathrm{HbCPK} 12$ HbCPK19 $\mathrm{HbCPK} 27$ HbCPK5 $\mathrm{HbCPK} 25$ HbCPK29 $\mathrm{HbCPK} 14$ HbCPK23 $\mathrm{HbCPK} 26$ HbCPK16 HbCPK7 $\mathrm{HbCPK} 9$ HbCPK2 HbCPK 4 $\mathrm{HbCPK} 11$ $\mathrm{HbCPK} 18$ HbCPK 15 $\mathrm{HbCPK} 10$ HbActin

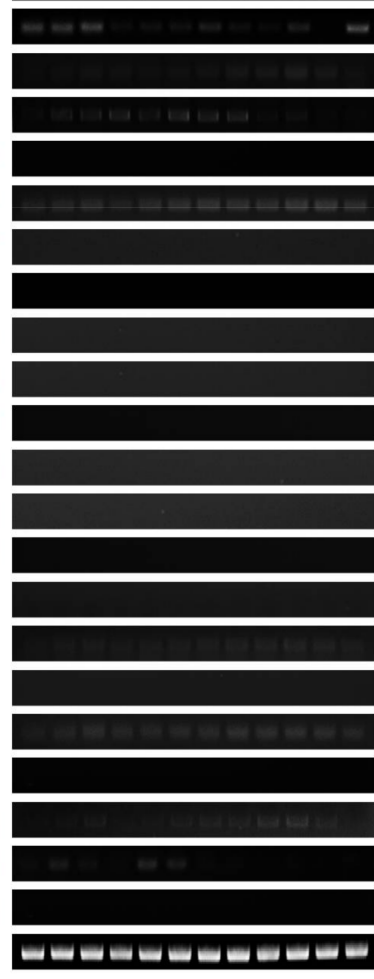

Figure 4. Expression profiles of the $30 \mathrm{CPK}$ genes in latex from three rubber tree varieties upon ethylene stimulation. (A) The heat map for $30 \mathrm{HbCPKs}$ at $0,3,12$ and $24 \mathrm{~h}$ under ethylene stimulate. The color bar shows at the bottom. The genes were ordered by the cluster of their expression patterns (left); (B) electrophoretogram of semi-quantitative PCR under different abiotic stresses. HbActin serves as the reference gene. 


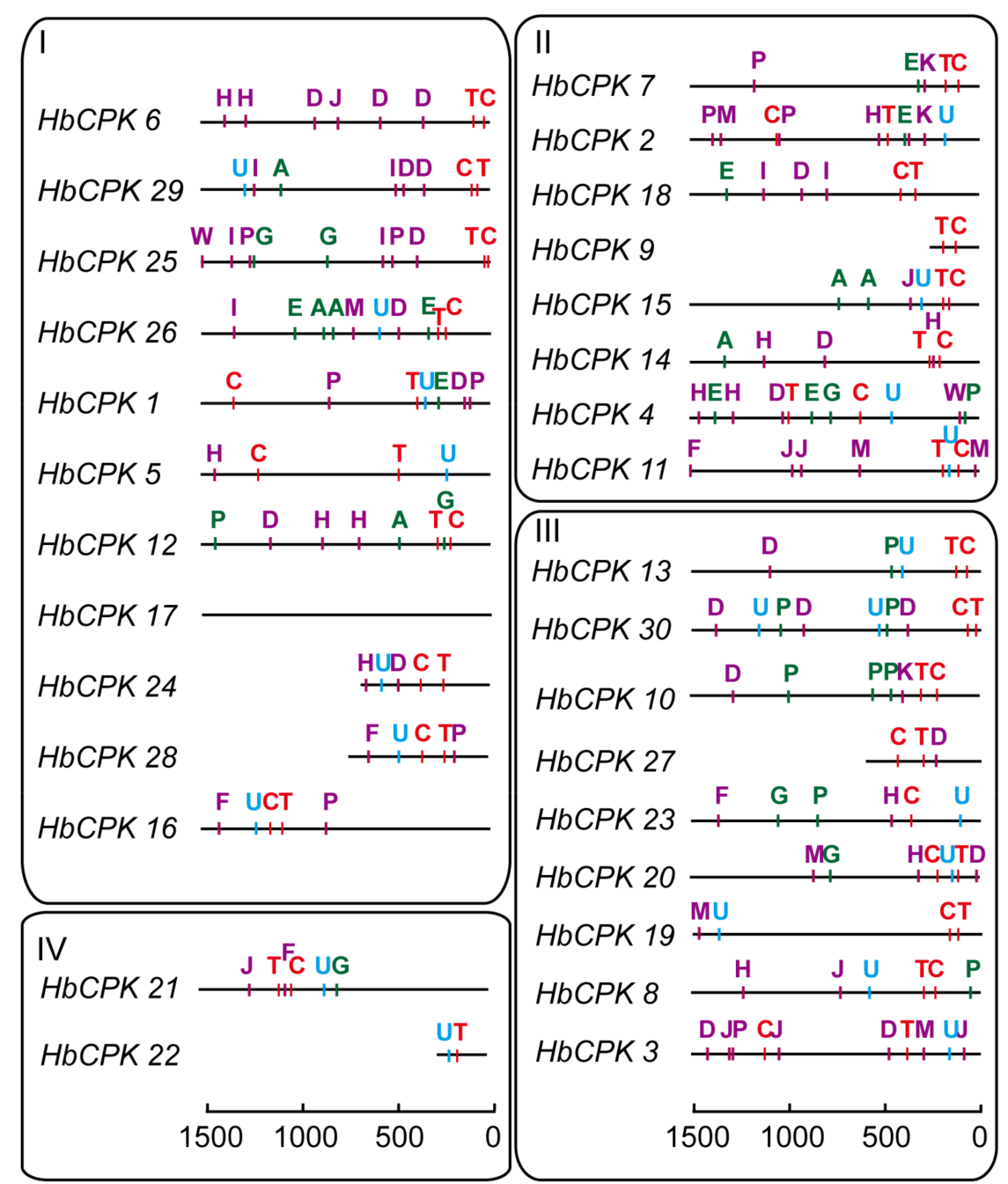

Figure 5. Distribution of cis-elements in $H b C P K$ gene promoter regions. The cis-elements of the $1.5 \mathrm{~kb}$ up-stream promoter regions of $H b C P K s$ were predicted using PlantCARE software. The $H b C P K s$ were divided into four groups according to the phylogenetic sub-families (Group I-IV). Colored capital letters represent different cis-elements at corresponding positions. Red, green, purple and blue letters represent transcription initiation, phytohormone response, stress response and high transcription classification of cis-elements, respectively. The detailed information for each cis-element is provided (Table S3).

\subsection{Protein Expression and PPI Analyses of Ethylene-Induced Proteins in Latex}

To investigate the protein expression pattern of $\mathrm{HbCPKs}$ after ethylene stimulation, our previous isobaric tags for relative and absolute quantification (iTRAQ) proteomic data [10] were re-analyzed using Hevea genome-derived protein database, identifying 3902 high-quality proteins ( $95 \%$ confidential peptides $\geq 2$; Table S4). Seven HbCPK members were detected, of which six (HbCPK12, HbCPK16, $\mathrm{HbCPK} 17, \mathrm{HbCPK} 18, \mathrm{HbCPK} 24$ and $\mathrm{HbCPK} 28$ ) were significantly induced by ethylene (fold change $\geq 2$; Table 3). The raw tandem mass spectrometry (MS/MS) spectra mapped to HbCPK proteins were provided (Figure S5). The representative signal intensities of iTRAQ report groups of seven identified $\mathrm{HbCPK}$ members were shown (Figure 6). These data further demonstrated that $\mathrm{HbCPK}$ members could be significantly induced upon ethylene treatment not only in transcript level but also in protein level. 
Table 3. Identification and quantitative information of seven detected $\mathrm{HbCPKs.}$

\begin{tabular}{|c|c|c|c|c|c|}
\hline \multirow{2}{*}{ НbСРК } & Peptide & $\mathrm{H}_{2} \mathrm{O}-48 \mathrm{~h}$ & Eth-48 h & $\mathrm{H}_{2} \mathrm{O}-96 \mathrm{~h}$ & Eth-96 h \\
\hline & Numbers & (114) & (115) & (116) & (117) \\
\hline НbСРК9 & 14 & $1.0000 *$ & 1.0471 & 1.0186 & 1.0965 \\
\hline HbCРК12 & 2 & 1.0000 & 3.3712 & 1.1478 & 3.1326 \\
\hline НbСРК16 & 6 & 1.0000 & 4.0926 & 2.1478 & 4.4463 \\
\hline НbСРК17 & 9 & 1.0000 & 9.7351 & 1.2985 & 9.1039 \\
\hline НbСРК18 & 7 & 1.0000 & 2.3335 & 0.8017 & 2.1677 \\
\hline $\mathrm{HbCPK} 24$ & 8 & 1.0000 & 13.9211 & 1.6346 & 12.5966 \\
\hline НbСРК28 & 6 & 1.0000 & 4.1687 & 1.7061 & 5.2481 \\
\hline
\end{tabular}

* Relative protein expression levels are defined as the ratio of 114/114, 115/114, 116/114 and 117/114. Eth, ethylene.
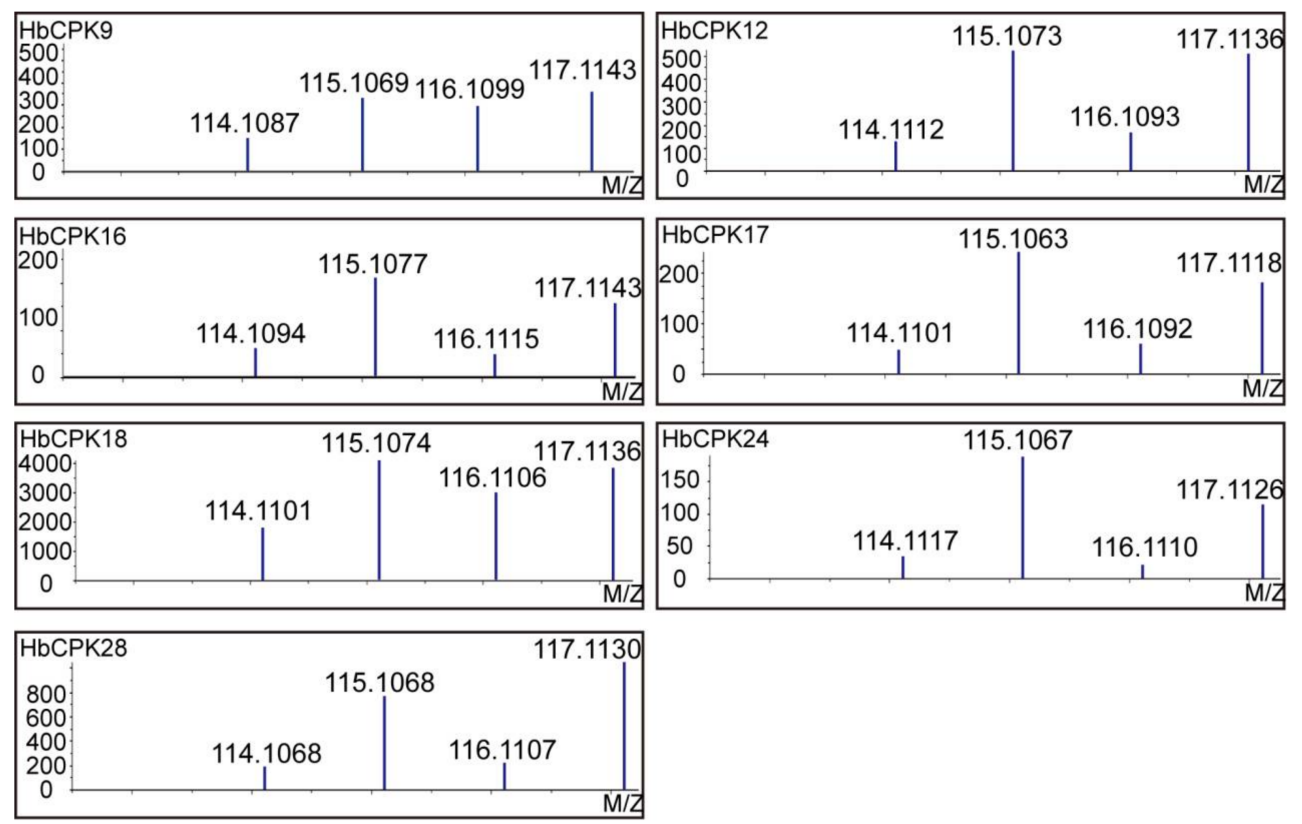

Figure 6. Proteomic validation of $H b C P K$ expression patterns in rubber tree latex before and after ethephon treatment. Representative mass spectra indicating the signal intensities of isobaric tags for the validated CPKs. The spectra correspond to the peptides of "SGYITMEELEHALK", "LSRLK", "LYQGYTQPEEQSTASHTKR", "IGQGQFGTTYLCTNK", "GQFGVTYLVTHK", "QATSSSSSTTKPANTVLPYQTSR" and "IIVGVVEACHSLGVMHR" that mapped to HbCPK9, HbCPK12, HbCPK16, HbCPK17, HbCPK18, HbCPK24 and HbCPK28, respectively.

Further comparative proteomics analysis of iTRAQ data resulted in 492 significantly different abundant proteins (DAPs). Protein-protein interaction analysis of these DAPs and 59 previously reported phosphorylated proteins [10] was performed using STRING 10.5. The result exhibited five PPI network clusters which were shadowed with different colors in Figure 7. Notably, protein kinases, especially calcium-dependent protein kinases, possess most key nodes of the interaction network (pink circles in Figure 7), implying the crucial roles of kinase proteins and protein phosphorylation in EIRP. These data for the first time validated the importance of HbCPK members in EIRP at protein level. 


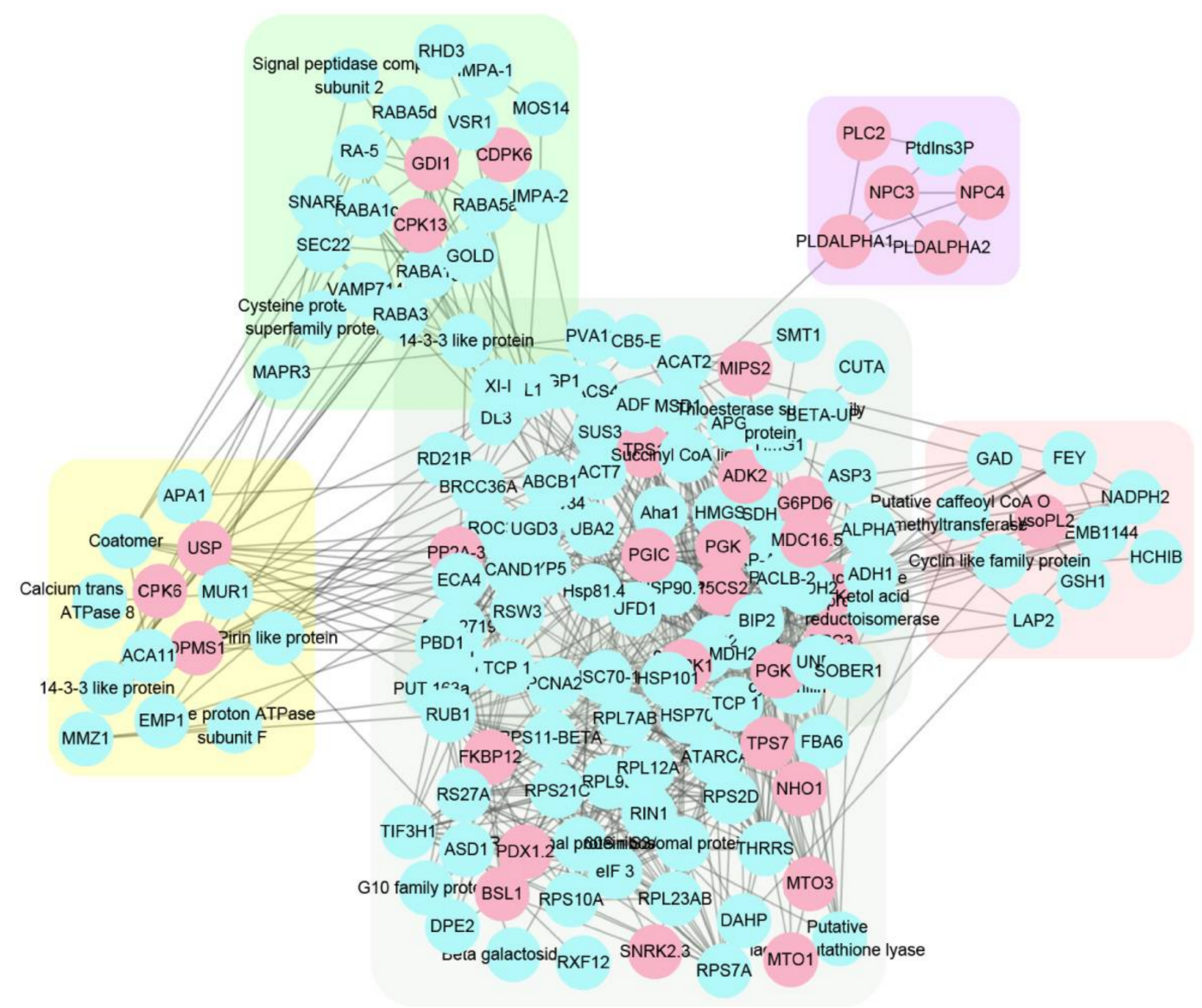

Figure 7. PPI networks of ethylene-regulated latex proteins and ethylene-responsible phosphorylated proteins. The 492 ethylene-regulated and 59 phosphorylated proteins identified were analyzed using the STRING database 10.5 with a medium confidence (0.400). Blue circles represent individual PPI network nodes with protein names. Pink circles indicate protein kinases. Five network clusters were shadowed by different colors.

\section{Discussion}

Ethylene stimulation is a widely used technological that can improve nature latex production [23]. Paradoxically, the expression levels of genes that known to involve in rubber biosynthesis are not upregulated upon ethylene stimulation. Most rubber latex biosynthesis related proteins, such as farnesyl diphosphate synthase [24], rubber elongation factor and small rubber particle protein [25], are not induced by ethylene stimulation, indicating that post-translational modification might be important for ethylene treatment response in rubber tree. Our previous works discovered that phosphorylation level of the latex proteins, and the expression level of calcium-dependent protein kinase was significantly increased after ethylene stimulation [10]. Xiao et al. identified $30 \mathrm{HbCPKs}$ and analyzed their expression patterns in Hevea and five other plant species using Solexa sequencing data, however, the transcript and protein expression levels of CPK family members in response to ethylene treatment were not thoroughly analyzed [21]. Hence, we performed a thoroughly investigation on the gene expression pattern and PPI network of $C P K$ members after ethylene treatment. Our study provided new evidence to support the hypothesis that $H b C P K$ is involved in the ethylene induced rubber latex production.

Gene duplication may cause functional diversity and further altered expression profiles and protein properties, and is a major evolutionary driver for helping plant species to meet a broader array of lineage-specific requirements [26]. The numbers of 3, 9, 2, 10 duplication events were found in soybean, rice, grape, and maize genome [17-20]. Analysis of $\mathrm{Ka} / \mathrm{Ks}$ selection pressure showed that 
all $\mathrm{HbCPK}$ paralogues were under purifying selection (Table 1), suggesting that the conservation of $H b C P K$ proteins might be crucial for their functions plant development. The expansion of a gene family within a genome could occur through gene functional divergence after duplication events. The HbCPK9 / HbCPK18 and HbCPK19/HbCPK20 duplication pairs had various expression patterns in different tissues and after ethylene stimulation, as well as different cis-elements distribution patterns (Figures 3-5), providing further evidence of the accelerated evolutionary rate of these two paralogues in rubber tree. As shown, $\mathrm{HbCPK20}$ was mainly up-regulated during ethylene stimulate in the latex of PR107 (high ethylene sensitivity cultivar) while HbCPK19 was not (Figure 4), suggesting that HbCPK19 might play the same role as its Arabidopsis orthologue AtCPK13 (regulating the stomatal aperture) [27] and $\mathrm{HbCPK} 20$ might gain new functions in EIRP of rubber tree.

The expression levels of some genes were found to be inconsistent in latex and $0 \mathrm{~h}$ ethylene treated latex, which may be caused by different experimental materials used in Figures 3 and 4. Similar results were also reported by Xiao et al. [21]. However, this difference in expression levels does not affect the analysis on $H b C P K S$ response to ethylene stimulation. Besides, $H b C P K 1,2,4,7,18$ and 26 had one or two ethylene responsive cis-element (Figure 5), but the expression levels of these genes were not induced by ethylene stimulation (Figure 4). The inconsistency between cis-element predicted by software and the regulation of the gene expression was reported by many researches [28,29]. It suggested that our understanding of the regulation mechanism of gene expression is still poor and the cis-elements predicted by the sequence information may have no function in many cases.

Introduction of genome predicated protein database to analyze our previous proteomic data greatly improved the number of identified proteins from 1596 to 3902 (Table S4). For the first time, seven $\mathrm{HbCPK}$ members were identified at protein level with raw spectra provided, in which HbCPK12, 16, 17, 18, 24 and 28 were significantly induced by ethylene (Table 3, Figures 6 and S5), but the expression of HbCPK12, 16 and 18 were not induced in mRNA levels after ethylene stimulation (Figure 4). Generally, frequent transcriptome and high mRNA stability can improve protein accumulation. However, many factors participate in the processes from transcription to translation and affect the translation efficiency of a given mRNA. The poly (A) tail length of the mRNA affects transcript stability and is closely correlated with translational efficiency [30,31]. This phenomenon could partly explain the different expression changes in response to ethylene treatment at cDNA and protein level we observed in Figures 4 and 6 . The fact that protein kinases possess the most key nodes in the PPI network suggested that ethylene signaling promotes the natural rubber production mainly through phosphorylation of functional proteins, but not through increasing the accumulation of functional enzymes (Figure 7). These proteomic analyses provide an explanation of the phenomenon that natural rubber biosynthesis related proteins are not induced by ethylene signal.

In summary, our research provides a comprehensive evolutionary and syntenic analysis of $\mathrm{HbCPK}$ members, suggesting that all $\mathrm{HbCPKS}$ were under purifying selection pressure and two duplication paralogues had an accelerated evolutionary rate. The expression profiling of $H b C P K$ family demonstrated that the ethylene-induced $H b C P K$ members have potential diverse functions in EIRP in three different ethylene-sensitivity rubber tree cultivars. The PPI network of DAPs revealed that protein kinases and phosphorylated proteins were involved in the key nodes of the ethylene-induced protein interaction network clusters, indicating the potential interaction among protein kinases and protein phosphorylation regulated upon ethylene stimulation in latex. This work provides a more comprehensive understanding of the $H b C P K s$ in ethylene induced rubber production improvement and potential transgenic targets for future molecular breeding of rubber tree for improving natural rubber production. 


\section{Materials and Methods}

\subsection{Identification and Sequence Analyses of CPK Gene Family in Rubber}

Protein sequences of CPK family members in Arabidopsis (Arabidopsis thaliana) and rice (Oryza sativa) were used for query [12,17]. Local BLASTP search was performed to identify CPK members in rubber tree genome [32]. All the remaining CPK candidates were further verified by InterProScan server (http: / / www.ebi.ac.uk/interpro/) to assess the existence of protein kinase domain (PF00069) and EF-hand_7 domain (PF13499). A carefully manual review of these sequences was carried out to amend the underlying mistake of rubber tree genome database.

The genomic location of $\mathrm{HbCPKs}$ was performed using local BLASTN against rubber tree genome database. Mapinspect was used to draw the location of CPKs in different scaffolds. The amino acid sequences of CPKs in rubber tree, Arabidopsis, rice, and a related-species cassava were used to construct phylogenetic tree by MEGA 5.0 (Hachioji, Tokyo, Japan) using neighbor-joining method with 1000 bootstrap test. Multiple sequence alignment was conducted using ClustalX 2.0 (Belfield, Dublin, Ireland) with default parameters to determine the conserved domain of CPKs. Multiple Em for Motif Elicitation (MEME) online software (http://meme-suite.org/tools/meme) was used to identify the conserved motifs of HbCPK members. The molecular weight and isoeletric points of HbCPKs were predicted by ExPASy server (http:/ / expasy.org/). Softberry (http:/ /linux1.softberry.com/berry.phtml) was used to predicted subcellular location of $\mathrm{HbCPK}$ members.

\subsection{Gene Structure, Duplication Event and Syntenic Analysis}

Gene structure was analyzed using GSDS server (http://gsds.cbi.pku.edu.cn/) based on the open reading farm (ORF) against $H b C P K s$ and $M e C P K s$ genomic sequences. Paralogues was determined by multiple sequence alignment with the identification $>90 \%$ [33]. DnaSP 5.0 software (Barcelona, Catalonia, Spain) was used to calculate $K a$ (nonsynonymous substitution rate) and Ks (synonymous substitution rate). The $\mathrm{Ka} / \mathrm{Ks}$ ratios for the $\mathrm{HbCPK}$ paralogues were calculated to assess the selection pressure; the ratio $>1,<1$, or $=1$ indicates positive, negative or neutral evolution, respectively [29]. Tajima relative rate tests [34] were analyzed by MEGA 5.0 using the amino acid sequences of the duplicated $H b C P K$ pairs. The syntenic relationships of paralogues and/or orthologues among Arabidopsis, cassava and rubber tree were analyzed using the Circos program [35].

\subsection{Plant Material and Ethylene Treatment}

Rubber trees of the three cultivars, named Reyan8-79 (high yielding, low ethylene sensitivity), Reyan7-33-97 (medium yielding, medium ethylene sensitivity) and PR107 (low yielding, high ethylene sensitivity) were planted in the experimental farm of Chinese Academy of Tropical Agricultural Sciences in Hainan Province in China. Different tissues, including roots, stems, leaves and untreated latex were collected from 10-year-old mature trees. Ethylene treated latex were collected from these three cultivars $0,3,12$ and $24 \mathrm{~h}$ after application of $1.5 \%$ ethephon. All materials were frozen in liquid nitrogen immediately after collected and stored at $-80^{\circ} \mathrm{C}$.

\subsection{Gene Expression Profiling of $\mathrm{HbCPKs}$}

Total RNAs were extracted using RNAprep pure plant kit (TIANGEN, Beijing, China) from different rubber tree materials, and $1 \mu \mathrm{g}$ of total RNA was used for first-strand cDNA synthesis. The gene-specific primers for qRT-PCR were designed using the software Primer Primer 5.0 (Davis, CA, USA) according to $\mathrm{HbCPK}$ gene sequences listed in Supplementary Table S1. Rubber tree actin gene (GenBank Acc. HQ260674.1) was used as an internal control. Quantitative real-time PCR (qRT-PCR) was conducted using Mx3005P real-time PCR system with SYBR green master mix (2X) (Thermo Fisher Scientific, Foster, CA, USA) in accordance with previously described PCR conditions [36]. Data were processed using $2^{-\Delta \Delta C t}$ methods, and the relative expression level of each $C P K$ gene were used to generate a heat map using MultiExperiment viewer software $(\mathrm{MeV}$, 
version 4.9, Boston, MA, USA). Semi-quantitative PCR assays were performed using the same cDNA templates and primers used for qRT-PCR. The expression levels of each $H b C P K$ gene were visualized using agarose gel electrophoresis of the corresponding PCR products.

\subsection{Cis-Element Distribution Analysis in HbCPK Promoter Regions}

The $1500 \mathrm{bp}$ sequence of the genomic sequence upstream to the start codon of each gene was obtained from genome database to investigate the cis-element distribution in $H b C P K$ promoter regions. These sequences were then submitted to PlantCARE server [37] to predict putative cis-element.

\subsection{Interaction Network Analysis of Ethylene-Induced Proteins in Latex}

The ethylene-induced and phosphorylated latex proteins were obtained by re-analysis of comparative proteomics data of rubber latex after ethylene treatment, using Proteinpilot 5.0 (Redwood City, CA, USA) and Hevea genome-derived protein database [10,30]. Only proteins with $95 \%$ confidential peptides $\geq 2$ were considered as identified (Proteinpilot score $\geq 1.3$ ). The PPI network was constructed by Search Tool for the Retrieval of Interacting Genes (STRING10.5) [38] with the confidence score $\geq 0.400$, and further visualized using Cytoscape 3.6.0 [39].

Supplementary Materials: Supplementary materials can be found at http:/ /www.mdpi.com/1422-0067/19/4/947/ s1.

Acknowledgments: This work was supported by grants of National Natural Science Foundation of China (Grant number 31570301), the Scientific and Technological Achievement Transformation Project of Bingtuan (Grant number 2016AC017), the International Science and Technology Cooperation Project of Shihezi University (Grant number GJHZ201708), and the Central Public-interest Research Fund for CATAS (No. 1630052016003, 1630052017006-9).

Author Contributions: Liping Zhu, Xiang Jin and Quanliang Xie performed the experiments; Liping Zhu and Qi Yao analyzed the data; Liping Zhu and Xiang Jin wrote the paper; Hongbin Li and Xuchu Wang conceived and designed the experiments.

Conflicts of Interest: The authors declare no conflict of interest.

\section{References}

1. Cornish, K. Similarities and differences in rubber biochemistry among plant species. Phytochemistry 2001, 57, 1123-1134. [CrossRef]

2. Liu, J.P.; Zhuang, Y.F.; Guo, X.L.; Li, Y.J. Molecular mechanism of ethylene stimulation of latex yield in rubber tree (Hevea brasiliensis) revealed by de novo sequencing and transcriptome analysis. BMC Genom. 2016, $17,257$. [CrossRef] [PubMed]

3. Dusotoit-Coucaud, A.; Kongsawadworakul, P.; Maurousset, L.; Viboonjun, U.; Brunel, N.; Pujade-Renaud, V.; Chrestin, H.; Sakr, S. Ethylene stimulation of latex yield depends on the expression of a sucrose transporter (HbSUT1B) in rubber tree (Hevea brasiliensis). Tree Physiol. 2010, 30, 1586-1598. [CrossRef] [PubMed]

4. Tungngoen, K.; Kongsawadworakul, P.; Viboonjun, U.; Katsuhara, M.; Brunel, N.; Sakr, S.; Narangajavana, J.; Chrestin, H. Involvement of HbPIP2;1 and HbTIP1;1 aquaporins in ethylene stimulation of latex yield through regulation of water exchanges between inner liber and latex cells in Hevea brasiliensis. Plant Physiol. 2009, 151, 843-856. [CrossRef] [PubMed]

5. Amalou, Z.; Gibrat, R.; Brugidou, C.; Trouslot, P.; d'Auzac, J. Evidence for an amiloride-inhibited Mg/2H antiporter in lutoid (Vacuolar) vesicles from latex of Hevea brasiliensis. Plant Physiol. 1992, 100, $255-260$. [CrossRef] [PubMed]

6. Dusotoit-Coucaud, A.; Brunel, N.; Kongsawadworakul, P.; Viboonjun, U.; Lacointe, A.; Julien, J.L.; Chrestin, H.; Sakr, S. Sucrose importation into laticifers of Hevea brasiliensis, in relation to ethylene stimulation of latex production. Ann. Bot. 2009, 104, 635-647. [CrossRef] [PubMed]

7. Duan, C.; Rio, M.; Leclercq, J.; Bonnot, F.; Oliver, G.; Montoro, P. Gene expression pattern in response to wounding, methyl jasmonate and ethylene in the bark of Hevea brasiliensis. Tree Physiol. 2010, 30, 1349-1359. [CrossRef] [PubMed] 
8. Pramoolkit, P.; Lertpanyasampatha, M.; Viboonjun, U.; Kongsawadworakul, P.; Chrestin, H.; Narangajavana, J. Involvement of ethylene-responsive microRNAs and their targets in increased latex yield in the rubber tree in response to ethylene treatment. Plant Physiol. Biochem. 2014, 84, 203-212. [CrossRef] [PubMed]

9. Yeang, H.Y.; Arif, S.M.; Yusof, F.; Sunderasan, E. Allergenic proteins of natural rubber latex. Methods 2002, 27, 32-45. [CrossRef]

10. Wang, X.; Wang, D.; Sun, Y.; Yang, Q.; Chang, L.; Wang, L.; Meng, X.; Huang, Q.; Jin, X.; Tong, Z. Comprehensive proteomics analysis of laticifer latex reveals new insights into ethylene stimulation of natural rubber production. Sci. Rep. 2015, 5, 13778. [CrossRef] [PubMed]

11. Harper, J.F.; Sussman, M.R.; Schaller, G.E.; Putnam-Evans, C.; Charbonneau, H.; Harmon, A.C. A calcium-dependent protein kinase with a regulatory domain similar to calmodulin. Science 1991, 252, 951-954. [CrossRef] [PubMed]

12. Cheng, S.H.; Willmann, M.R.; Chen, H.C.; Sheen, J. Calcium signalling through protein kinases. The Arabidopsis calcium-dependent protein kinase gene family. Plant Physiol. 2002, 129, 469-485. [CrossRef] [PubMed]

13. Klimecka, M.; Muszyńska, G. Structure and functions of plant calcium-dependent protein kinases. Acta Biochim. Pol. 2007, 54, 219-233. [PubMed]

14. Harmon, A.C. Calcium-regulated protein kinases of plants. Gravit. Space Biol. Bull. 2003, 16, 83-90. [PubMed]

15. Hu, W.; Hou, X.; Xia, Z.; Yan, Y.; Wei, Y.; Wang, L.; Zou, M.; Lu, C.; Wang, W.; Peng, M. Genome-wide survey and expression analysis of the calcium-dependent protein kinase gene family in cassava. Mol. Genet. Genom. 2016, 291, 241-253. [CrossRef] [PubMed]

16. Ranty, B.; Aldon, D.; Cotelle, V.; Galaud, J.P.; Thulea, P.; Mazars, C. Calcium sensors as key hubs in plant responses to biotic and abiotic stresses. Front. Plant Sci. 2016, 7, 327. [CrossRef] [PubMed]

17. Ray, S.; Agarwal, P.; Arora, R.; Kapoor, S.; Tyagi, A.K. Expression analysis of calcium-dependent protein kinase gene family during reproductive development and abiotic stress conditions in rice (Oryza sativa L. ssp. indica). Mol. Genet. Genom. 2007, 278, 493-505. [CrossRef] [PubMed]

18. Kong, X.; Lv, W.; Jiang, S.; Zhang, D.; Cai, G.; Pan, J.; Li, D. Genome-wide identification and expression analysis of calcium-dependent protein kinase in maize. BMC Genom. 2013, 14, 433. [CrossRef] [PubMed]

19. Zhang, K.; Han, Y.T.; Zhao, F.L.; Hu, Y.; Gao, Y.R.; Ma, Y.F.; Zheng, Y.; Wang, Y.J.; Wen, Y.Q. Genome-wide identification and expression analysis of the CDPK gene family in grape, Vitis spp. BMC Plant Biol. 2015, 15, 164. [CrossRef] [PubMed]

20. Liu, H.; Che, Z.; Zeng, X.; Zhou, X.; Sitoe, H.M.; Wang, H.; Yu, D. Genome-wide analysis of calciumdependent protein kinases and their expression patterns in response to herbivore and wounding stresses in soybean. Funct. Integr. Genom. 2016, 16, 481-493. [CrossRef] [PubMed]

21. Xiao, X.H.; Yang, M.; Sui, J.L.; Qi, J.Y.; Fang, Y.J.; Hu, S.N.; Tang, C.R. The calcium-dependent protein kinase $(C D P K)$ and CDPK-related kinase gene families in Hevea brasiliensis-comparison with five other plant species in structure, evolution, and expression. FEBS Open 2016, 7, 4-24. [CrossRef] [PubMed]

22. Zhu, X.; Wang, M.; Li, X.; Jiu, S.; Wang, C.; Fang, J. Genome-wide analysis of the sucrose synthase gene family in grape (Vitis vinifera): Structure, evolution, and expression profiles. Genes 2017, 8, 111. [CrossRef] [PubMed]

23. Putranto, R.A.; Herlinawati, E.; Rio, M.; Leclercq, J.; Piyatrakul, P.; Gohet, E.; Sanier, C.; Oktavia, F.; Pirrello, J.; Montoro, P. Involvement of ethylene in the latex metabolism and tapping panel dryness of Hevea brasiliensis. Int. J. Mol. Sci. 2015, 16, 17885-17908. [CrossRef] [PubMed]

24. Adiwilaga, K.; Kush, A. Cloning and characterization of cDNA encoding farnesyl diphosphate synthase from rubber tree (Hevea brasiliensis). Plant Mol. Biol. 1996, 30, 935-946. [CrossRef] [PubMed]

25. Tong, Z.; Wang, D.; Sun, Y.; Yang, Q.; Meng, X.; Wang, L.; Feng, W.; Li, L.; Wurtele, E.S.; Wang, X. Comparative proteomics of rubber latex revealed multiple protein species of REF/SRPP family respond diversely to ethylene stimulation among different rubber tree clones. Int. J. Mol. Sci. 2017, 18, 958. [CrossRef] [PubMed]

26. Hamel, L.P.; Sheen, J.; Séguin, A. Ancient signals: Comparative genomics of green plant CDPKs. Trends Plant Sci. 2014, 19, 79-89. [CrossRef] [PubMed]

27. Ronzier, E.; Corratgé-Faillie, C.; Sanchez, F.; Prado, K.; Brière, C.; Leonhardt, N.; Thibaud, J.B.; Xiong, T.C. CPK13, a noncanonical $\mathrm{Ca}^{2+}$-dependent protein kinase, specifically inhibits KAT2 and KAT1 shaker $\mathrm{K}^{+}$ channels and reduces stomatal opening. Plant Physiol. 2014, 166, 314-326. [CrossRef] [PubMed] 
28. Kong, F.; Wang, J.; Cheng, L.; Liu, S.; Wu, J.; Peng, Z.; Lu, G. Genome-wide analysis of the mitogen-activated protein kinase gene family in Solanum lycopersicum. Gene 2012, 499, 108-120. [CrossRef] [PubMed]

29. Jin, X.; Zhu, L.; Yao, Q.; Meng, X.; Ding, G.; Wang, D.; Xie, Q.; Tong, Z.; Tao, C.; Yu, L.; et al. Expression profiling of mitogen-activated protein kinase genes reveals their evolutionary and functional diversity in different rubber tree (Hevea brasiliensis) cultivars. Genes 2017, 8, 261. [CrossRef] [PubMed]

30. De Sousa Abreu, R.; Penalva, L.O.; Marcotte, E.M.; Vogel, C. Global signatures of protein and mRNA expression levels. Mol. Biosyst. 2009, 5, 1512-1526. [CrossRef] [PubMed]

31. Tuller, T.; Kupiec, M.; Ruppin, E. Determinants of protein abundance and translation efficiency in S. cerevisiae. PLoS Comput. Biol. 2007, 3, e248. [CrossRef] [PubMed]

32. Tang, C.; Yang, M.; Fang, Y.; Luo, Y.; Gao, S.; Xiao, X.; An, Z.; Zhou, B.; Zhang, B.; Tan, X.; et al. The rubber tree genome reveals new insights into rubber production and species adaptation. Nat. Plants 2016, 2, 16073. [CrossRef] [PubMed]

33. Tao, C.; Jin, X.; Zhu, L.; Xie, Q.; Wang, X.; Li, H. Genome-wide investigation and expression profiling of APX gene family in Gossypium hirsutum provide new insights in redox homeostasis maintenance during different fiber development stages. Mol. Genet. Genom. 2018. [CrossRef] [PubMed]

34. Tajima, F. Unbiased estimation of evolutionary distance between nucleotide sequences. Mol. Biol. Evol. 1993, 10, 677-688. [PubMed]

35. Krzywinski, M.; Schein, J.; Birol, I.; Connors, J.; Gascoyne, R.; Horsman, D.; Jones, S.J.; Marra, M.A. Circos: An information aesthetic for comparative genomics. Genome Res. 2009, 19, 1639-1645. [CrossRef] [PubMed]

36. Jin, X.; Wang, L.; He, L.; Feng, W.; Wang, X. Two-dimensional gel electrophoresis-based analysis provides global insights into the cotton ovule and fiber proteomes. Sci. China Life Sci. 2016, 59, 154-163. [CrossRef] [PubMed]

37. Xin, S.; Tao, C.; Li, H. Cloning and functional analysis of the promoter of an ascorbate oxidase gene from Gossypium hirsutum. PLoS ONE 2016, 11, e0161695. [CrossRef] [PubMed]

38. Szklarczyk, D.; Morris, J.H.; Cook, H.; Kuhn, M.; Wyder, S.; Simonovic, M.; Santos, A.; Doncheva, N.T.; Roth, A.; Bork, P.; et al. The STRING database in 2017: Quality-controlled protein-protein association networks, made broadly accessible. Nucleic Acids Res. 2017, 45, 362-368. [CrossRef] [PubMed]

39. Shannon, P.; Markiel, A.; Ozier, O.; Baliga, N.S.; Wang, J.T.; Ramage, D.; Amin, N.; Schwikowski, B.; Ideker, T. Cytoscape: A software environment for integrated models of biomolecular interaction networks. Genome Res. 2003, 13, 2498-2504. [CrossRef] [PubMed] 